\title{
Preparation of $\gamma$-PGA/chitosan composite tissue engineering matrices
}

\author{
Chien-Yang Hsieh ${ }^{\mathrm{a}}$, Sung-Pei Tsai ${ }^{\mathrm{a}}$, Da-Ming Wang ${ }^{\mathrm{a}}$, Yaw-Nan Chang ${ }^{\mathrm{b}}$, \\ Hsyue-Jen Hsieh ${ }^{\mathrm{a}, *}$ \\ ${ }^{a}$ Department of Chemical Engineering, National Taiwan University, Taipei 10617, Taiwan, ROC \\ ${ }^{\mathrm{b}}$ Department of Bioindustry Technology, Da-Yeh University, Changhua, Taiwan, ROC
}

Received 27 October 2004; accepted 8 February 2005

Available online 7 April 2005

\begin{abstract}
$\gamma$-poly(glutamic acid) ( $\gamma$-PGA), a hydrophilic and biodegradable polymer, was chosen to modify chitosan matrices to produce a $\gamma$ PGA/chitosan composite biomaterial. Three types of both dense and porous composite matrices containing different amounts of $\gamma$ PGA were fabricated. Chitosan and $\gamma$-PGA matrices were also prepared as controls. Fluorescence staining indicated that chitosan and $\gamma$-PGA were evenly distributed in the composite matrices. SEM micrographs showed that an interconnected porous structure with a pore size of $30-100 \mu \mathrm{m}$ was present in all porous matrices except the $\gamma$-PGA ones. By increasing the percentage of $\gamma$-PGA from $0 \%$ to $20 \%$, the swelling ratio of the matrices was enhanced from 1.6 to 3.2. Similarly, the contact angle of the matrices decreased from $113^{\circ}$ to $94^{\circ}$. These data suggested that the surface hydrophilicity, water absorption rate, and swelling ratio were improved by adding $\gamma$-PGA to the matrices. Additionally, the mechanical strength of the porous $\gamma$-PGA/chitosan matrices was about $25-50 \%$, higher than that of the unmodified chitosan matrices. The composite matrices were also examined and found to be an appropriate environment for cell attachment and proliferation. The cell density on the $20 \% \gamma$-PGA-modified matrices was almost triple that on the unmodified chitosan matrices on day 5. In summary, the $\gamma$-PGA/chitosan composite matrices, due to their better hydrophilic, cytocompatible, and mechanical properties, are very promising biomaterials for tissue engineering applications.
\end{abstract}

(C) 2005 Elsevier Ltd. All rights reserved.

Keywords: Chitosan; $\gamma$-PGA; Tissue engineering; Composite matrices

\section{Introduction}

Chitosan is a polysaccharide constituted by $N$ glucosamine and $N$-acetyl-glucosamine units (Fig. 1A), in which the number of $\mathrm{N}$-glucosamine units exceeds $50 \%$ [1]. Chitosan is positively charged and solubilized by protonation of its amino groups when the solution $\mathrm{pH}$ is below 6. Chitosan can be degraded into nontoxic products in vivo [2], and thus it has been widely used in various biomedical applications [3-10]. However, the poor hydrophilicity and cytocompatibility hinder the usage of chitosan in the field of tissue engineering.

In this study, $\gamma$-poly(glutamic acid) $(\gamma$-PGA), a hydrophilic and biodegradable polymer, was utilized

\footnotetext{
${ }^{*}$ Corresponding author. Tel.: 886223633097 ; fax: 886223623040.

E-mail address: hjhsieh@ntu.edu.tw (H.-J. Hsieh).
}

to enhance the hydrophilicity and cytocompatibility of chitosan-based biomaterials. $\gamma$-PGA is a polyamino acid formed by the amide bond linkage between the amino group on the $\alpha$ carbon of one glutamic acid and the carboxyl group on the $\gamma$ carbon of the next glutamic acid (Fig. 1B). $\gamma$-PGA is nontoxic, hydrophilic, and biodegradable, and has been successfully utilized in bioglue and drug delivery systems [11-17]. It has many carboxy groups that make it a polyanionic biopolymer. As mentioned previously, chitosan is a positively charged polyelectrolyte in an acidic environment. When a negatively charged polyelectrolyte such as alginate or $\gamma$-PGA is introduced, a polyion complex can be formed by electrostatic attraction at the interfaces $[18,19]$. This phenomenon was used to fabricated fibers and capsules in several previous studies [20-27]. However, it is quite difficult to obtain a homogeneous solution of $\gamma$-PGA 
and chitosan. When we tried to directly mix chitosan and $\gamma$-PGA solutions, polyion complexes immediately formed at the interfaces, inducing a huge aggregate. In order to obtain a homogeneous solution of chitosan and $\gamma$-PGA, we developed an alternative method, in which chitosan powder, not solution, was directly added into a $\gamma$-PGA solution with strong stirring (see Section 2.2). This method prevented the formation of polyion complexes at the interfaces and thus a homogeneous solution of chitosan and $\gamma$-PGA was obtained.

In the present study, homogeneous solutions containing different molar ratios of $\gamma$-PGA to chitosan were prepared and used to produce both dense and porous matrices. To analyze the distribution of $\gamma$-PGA and chitosan in the matrices, fluorescence staining was carried out. Changes in the hydrophilicity, swelling ratio, water uptake and retention abilities, and mechanical properties when adding $\gamma$-PGA to the matrices were also investigated. Our results indicated that the pore structure of the porous $\gamma$-PGA/ chitosan composite matrices was interconnected with a pore size of $30-100 \mu \mathrm{m}$. The addition of $\gamma$-PGA significantly enhanced the material properties of the chitosan matrix and made it more hydrophilic and cytocompatible, and thus more suitable for tissue engineering applications.

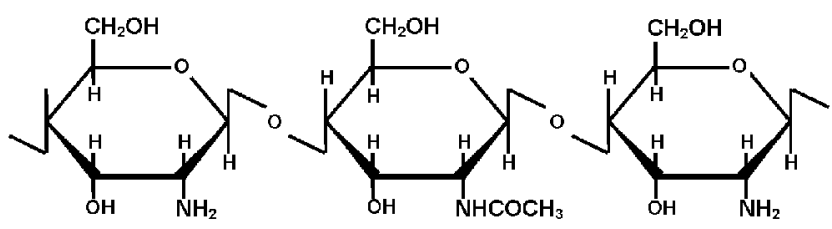

$\mathrm{N}$-glucosamine $\mathrm{N}$-acetylglucosamine

(A)

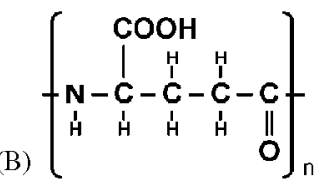

Fig. 1. (A) Structure of chitosan. Chitosan is constituted of two types of randomly distributed repeat units, $\mathrm{N}$-glucosamine and $\mathrm{N}$-acetylglucosamine, with the number of $N$-glucosamine units higher than $50 \%$. (B) Structure of $\gamma$-PGA.

\section{Materials and methods}

\subsection{Materials}

Chitosan (with a MW of about $4 \times 10^{5}$ and degree of deacetylation of $90 \%$ ) was purchased from Taiwan Chitin Chitosan Co. (Taipei, Taiwan). $\gamma$-PGA was provided by Prof. Yaw-Nan Chang of the Department of Bioindustry Technology, Da-Yeh University, Taiwan (with a MW of about $2 \times 10^{6}$ ). Other chemicals of the highest purity available were used. Materials for cell culture were purchased from Sigma-Aldrich (St. Louis, MO, USA), Biochrom AG (Berlin, Germany), Biological Industries (Kibbute, Israel), or GIBCO Invitrogen (Grand Island, NY, USA). Tissue culture flasks and 12well plates were obtained from Corning (Schiphol-Rijk, The Netherlands). The Micro BCA protein assay kit was purchased from Pierce (Rockford, IL, USA).

\subsection{Preparation of matrices}

Acetic acid was used as a solvent for both chitosan and $\gamma$-PGA. To avoid the polyion complex aggregation caused by the electrostatic attraction between chitosan and $\gamma$-PGA, an alternative method was developed. First, $\gamma$-PGA was dissolved in water by stirring to form a homogeneous solution. Then chitosan powder, not solution, was added with strong stirring to ensure that the powder was uniformly distributed in the $\gamma$-PGA solution. Then, acetic acid was added to the solution. Since the chitosan powder was already uniformly dispersed, the addition of acetic acid caused the chitosan powder to immediately dissolve, thus avoiding the aggregation caused by the $\gamma$-PGA/chitosan polyion complexes. Therefore, a homogeneous solution was obtained.

We divided the matrix solutions into five groups according to the ratio of the number of repeat unit on $\gamma$-PGA (P) to that on chitosan (C), namely C100, P1C99, P5C95, P20C80, and P100 (see Table 1). The reason for choosing the repeat unit ratio instead of the weight percent as the base for calculation was that the number of hydrogen bonds was a concern. There are two types of repeat units on chitosan: $\mathrm{N}$-glucosamine and $\mathrm{N}$ acetyl-glucosamine (Fig. 1A). Both units have functional groups which form hydrogen bonds ( $N$-glucosamine has

Table 1

Composition of and abbreviations for the five types of matrix solutions

\begin{tabular}{lllll}
\hline & C100 & P1C99 & P5C95 & P100 \\
\hline$\gamma$-PGA $(g)$ & 0 & 0.031 & 0.158 \\
Chitosan $(g)$ & 4 & 3.969 & 3.842 & 0.653 \\
Ratio of the number of repeat units $(\gamma$-PGA: chitosan) & $0: 100$ & $1: 99$ & 3.347 & 9 \\
0.2 M acetic acid solution $(g)$ & 96 & 96 & 96 \\
\hline
\end{tabular}


$-\mathrm{OH}$ and $-\mathrm{NH}_{2}$ while $\mathrm{N}$-acetyl-glucosamine has $-\mathrm{OH}$ and $-\mathrm{NHCOCH}_{3}$ ). The repeat unit of $\gamma$-PGA also has a functional group which forms hydrogen bonds ($\mathrm{COOH}$ ) (Fig. 1B). Additional interactions may occur between the positively charged amino group on the $\mathrm{N}$ glucosamine unit of chitosan and the negatively charged carboxyl group on the glutamic acid unit of $\gamma$-PGA. The total weight percentage of $\gamma$-PGA and chitosan in the matrix solution was $4 \%$. The compositions of all five types of matrix solutions are summarized in Table 1. For example, P1C99 indicates that the ratio of the number of repeat unit of $\gamma$-PGA to that of chitosan in the matrix solution is 1:99. Other matrices were similarly denoted.

We used the same matrix solutions but different methods to prepare both dense and porous matrices. For the preparation of dense matrices, each solution was prepared and then centrifuged for $15 \mathrm{~min}$ at $3000 \times \mathrm{g}$. The solution was then poured into dishes and dried in an oven for $24 \mathrm{~h}$ with the temperature maintained at $4{ }^{\circ} \mathrm{C}$. The dehydrated matrices were then immersed in a $3 \mathrm{M}$ $\mathrm{NaOH}$ solution for $12 \mathrm{~h}$, followed by rinsing with ethanol. The matrices were washed using a phosphatebuffered saline (PBS) solution. The matrices were dried again in the oven at $40^{\circ} \mathrm{C}$ for $24 \mathrm{~h}$ and then stored at $4{ }^{\circ} \mathrm{C}$ for further analyses.

For the preparation of porous matrices, the freezegelation method was used [28]. After centrifugation, the matrix solution was poured into dishes and frozen for $12 \mathrm{~h}$ with the temperature maintained at $-80^{\circ} \mathrm{C}$. The frozen samples were immersed in a $3 \mathrm{M} \mathrm{NaOH} /$ ethanol solution at $-20^{\circ} \mathrm{C}$ for $12 \mathrm{~h}$, followed by rinsing with ethanol. The matrices were then washed using the PBS solution.

\subsection{Scanning electron microscopy (SEM) analysis for porous matrices}

To investigate the microstructure, cross-sections of the porous matrices were observed using SEM. For SEM, matrix samples were rinsed in a $95 \%$ ethanol solution at $20^{\circ} \mathrm{C}$ for $12 \mathrm{~h}$ and then dried in a lyophilizer. Samples were coated with gold-palladium and then examined by SEM.

\subsection{Fluorescence microscopic observation of porous matrices}

To determine whether chitosan and $\gamma$-PGA were evenly distributed in the matrices, chitosan was conjugated using rhodamine B (rhodamine B isothiocynate, a fluorescent dye, with excitation at $510-550 \mathrm{~nm}$ and fluorescence emission of red light), and $\gamma$-PGA was conjugated using FITC (fluorescein isothiocynate, a fluorescent dye with excitation at $450-480 \mathrm{~nm}$ and fluorescence emission of green light) through a lysine/
EDC linking system [29-31]. The rhodamine B-labeled chitosan and FITC-labeled $\gamma$-PGA were used to prepare porous matrices as described in Section 2.2. Cryomicrotome sections of the matrices were then observed and photographed using a fluorescence microscope (Olympus IX70, with fluorescence attachment IX-FLA).

\subsection{Contact angles and swelling behavior of dense matrices}

Contact angles of the dense matrix surfaces were measured by the sessile method, using a video camera mounted on a microscope to record the droplet image. A droplet of $2 \mu \mathrm{L}$ was used in this experiment.

To measure the swelling behavior of the dense matrices, samples were sliced into $1 \times 4-\mathrm{cm}$ pieces, dried in an oven for $3 \mathrm{~h}$, and then vacuum-dried for $12 \mathrm{~h}$. The dry weights of the matrices $\left(W_{\text {dry }}\right)$ were immediately measured. Afterwards, the matrices were immersed in PBS maintained at $37^{\circ} \mathrm{C}$, and weighed at specific time points to determine the wet weights $\left(W_{\text {wet }}\right)$. The swelling ratios of the matrices were calculated using the following formula:

Swelling ratio $=\left(W_{\text {wet }}-W_{\text {dry }}\right) / W_{\text {dry }}$.

\subsection{Analysis of mechanical properties}

The mechanical properties of the dense and porous matrices were determined in a tensile strength instrument (model LRX, Lloyd, Hampshire, UK). The prepared matrices were swollen in PBS for $2 \mathrm{~h}$ and cut into a specific dog-bone shape $(6 \mathrm{~cm}$ long, $2 \mathrm{~cm}$ wide at the ends and $1 \mathrm{~cm}$ wide in the middle). The thickness of each individual matrix was measured. The mechanical analysis was performed at a stretching rate of $10 \mathrm{~mm} /$ min with a pre-load of $0.5 \mathrm{~N}$ to determine the maximum load for each matrix. Because of its high solubility in water, the mechanical properties of the $\gamma$-PGA matrix (P100) could not be measured.

\subsection{Adsorption of protein onto matrix surfaces}

The adsorption of serum proteins on different dense matrices was determined in 12-well plates. To each well was added $500 \mu \mathrm{L}$ fetal bovine serum solution $(5 \%$, diluted by PBS), which was then incubated at $37^{\circ} \mathrm{C}$ for $6 \mathrm{~h}$. Each well was washed with PBS, and then desorption buffer was introduced to the well for $10 \mathrm{~min}$. Desorption buffer contained $0.25 \mathrm{wt} \%$ sodium deoxycholate, $1 \mathrm{wt} \%$ NP-40, $0.2 \mathrm{wt} \%$ SDS, and $4 \mathrm{~mm}$ PMSF. The resulting desorption buffer containing desorbed proteins was recovered for a protein concentration assay using the BCA protein assay kit (Pierce). 


\subsection{Determination of cytocompatibility}

For cytocompatibility studies, dense matrices were prepared in 12-well plates as described in Section 2.2. The matrices were sterilized using a $70 \%$ ethanol solution for $24 \mathrm{~h}$. Rat osteosarcoma (ROS) cells cultured in MEM supplemented with $10 \%$ FBS were seeded onto various matrices at a density of $2 \times 10^{4}$ cells/well. To determine the cell numbers on various surfaces, a standard curve of cell number vs. respective protein concentration was first established. Then this standard curve was used to indirectly determine the cell number by measuring the protein concentration of a cell lysate. To carry out the measurement, cultured cells (on the matrices) were washed three times with PBS. Lysis buffer (the same as the desorption buffer in Section 2.7) was introduced to the cells with a reaction time of $10 \mathrm{~min}$. The resulting cell lysate was recovered for the protein concentration assay using the BCA protein assay kit (Pierce).

\section{Results and discussion}

\subsection{SEM analysis of porous matrices}

The microstructure of a matrix has a prominent influence on cell proliferation, function, and migration in tissue engineering. To observe and confirm the microstructure, the cross-section of porous matrices prepared by the freeze-gelation method was investigated using SEM (Fig. 2). The interconnected 3D porous structure was observed, and the mean pore size was about $30-100 \mu \mathrm{m}$ (estimated by direct measurement using SEM photography). Furthermore, with an increase in the amount of $\gamma$-PGA, the surface structures of the matrices became smoother, and less shrinkage was observed.
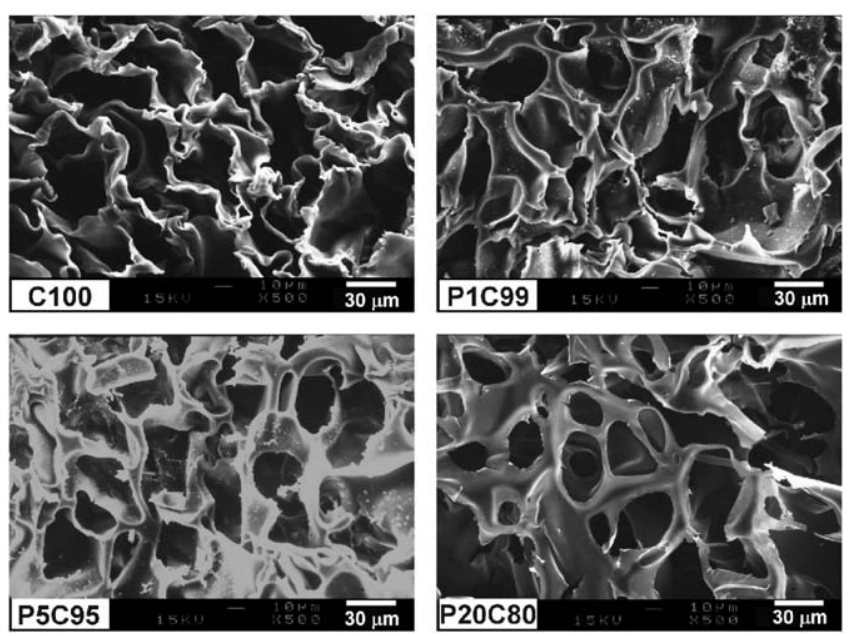

Fig. 2. SEM micrographs of the cross-section of chitosan (C100) and $\gamma$-PGA/chitosan (P1C99, P5C95, and P20C80) porous matrices.
According to the SEM micrographs, we demonstrated that a homogeneous solution of chitosan and $\gamma$-PGA can be made into porous matrices by the freeze-gelation method. Previous studies only demonstrated that chitosan and alginate or polyamino acid could be made into fibers and capsules by forming polyion complexes [27]. Herein we overcame the problem of forming polyion complexes at the solution interfaces, and successfully constructed porous and homogeneous $\gamma$-PGA/chitosan composite matrices (see Section 3.2).

\subsection{Fluorescence staining of chitosan and $\gamma-P G A$ in the porous matrices}

To determine whether chitosan and $\gamma$-PGA were evenly distributed in the matrices, fluorescent chitosan and $\gamma$-PGA were prepared and utilized to fabricate a composite matrix (P20C80). Cross-sections of the matrices were examined by fluorescence microscopy, and these selectively displayed the distribution of either chitosan or $\gamma$-PGA (Fig. 3).

The bright-field image (Fig. 3A) was very similar to the images of fluorescence staining of chitosan (Fig. 3B) and $\gamma$-PGA (Fig. 3C), indicating that chitosan and $\gamma$-PGA were uniformly distributed in the porous composite matrices.

\subsection{Contact angles and swelling behavior of dense matrices}

Hydrophilicity is an important characteristic property for biomaterials. To determine the hydrophilicity of the dense matrices, their contact angles and bulk water absorption abilities were investigated. Since the contact angle decreased with time due to water absorption, contact angle measurements were carried out three times
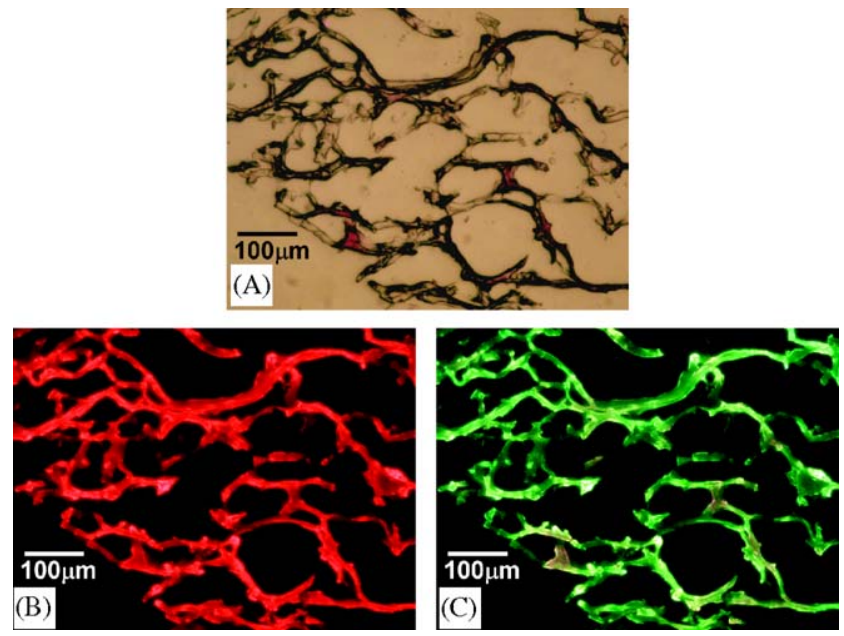

Fig. 3. Cross-sections of P20C80 porous matrices. (A) Bright-field micrograph, (B) fluorescence staining of chitosan, and (C) fluorescence staining of $\gamma$-PGA. Please see Section 2.4 for more details. 
Table 2

Water contact angles, wetting rates, and swelling ratios of the chitosan $(\mathrm{C} 100)$ and $\gamma$-PGA/chitosan $(\mathrm{P} 1 \mathrm{C} 99, \mathrm{P} 5 \mathrm{C} 95, \mathrm{P} 20 \mathrm{C} 80)$ surfaces $(n=7$, mean \pm SD)

\begin{tabular}{|c|c|c|c|c|c|}
\hline & \multicolumn{3}{|c|}{ Contact angle $\left(^{\circ}\right)$} & \multirow[t]{2}{*}{ Wetting rate $(\% / s)^{\mathrm{b}}$} & \multirow[t]{2}{*}{ Swelling ratio } \\
\hline & M. T. $(5 \mathrm{~s})^{\mathrm{a}}$ & M. T. (55s) & M. T. (115s) & & \\
\hline $\mathrm{C} 100$ & $113.4 \pm 0.93$ & $107.8 \pm 0.59$ & $104.3 \pm 1.06$ & $0.083 \pm 0.018$ & $1.65 \pm 0.08$ \\
\hline P1C99 & $105.6 \pm 0.43$ & $96.17 \pm 1.56$ & $89.24 \pm 1.14$ & $0.086 \pm 0.014$ & $2.03 \pm 0.06$ \\
\hline Р5C95 & $100.3 \pm 0.72$ & $88.81 \pm 1.35$ & $81.90 \pm 2.14$ & $0.167 \pm 0.026$ & $2.18 \pm 0.07$ \\
\hline P20C80 & $94.26 \pm 0.54$ & $84.51 \pm 0.84$ & $74.92 \pm 0.54$ & $0.176 \pm 0.010$ & $3.19 \pm 0.10$ \\
\hline
\end{tabular}

${ }^{\mathrm{a}} \mathrm{M}$. T.: measuring time.

${ }^{\mathrm{b}}$ Wetting rate, decrease in the contact angle per unit time $(\%)$.

with measuring times of 5,55 , and $115 \mathrm{~s}$, respectively for each matrix. The contact angle of the P20C80 composite matrix was $20^{\circ}-30^{\circ}$ smaller than that of the chitosan (C100) matrix, indicating that the addition of $\gamma$-PGA greatly improved the hydrophilicity of the chitosan matrix (Table 2). Additionally, by comparing the data at different measuring times, we found that the contact angles decreased with time, indicating that the hydrophilicity of the matrices increased with the absorption of water. To compare the speed at which water was absorbed by each matrix, we defined a "wetting rate" as the rate of decrease in the contact angle between 5 and $115 \mathrm{~s}$. It was found that the larger the $\gamma$-PGA amount, the higher the wetting rate would be (Table 2). The swelling ratio also increased from 1.6 for the chitosan (C100) matrix to 3.2 for the P20C80 composite matrix (Table 2). Taken together, the addition of $\gamma$-PGA greatly enhanced the hydrophilicity, wetting rate, and swelling ratio of the chitosan composite matrices, especially those of the P20C80 matrix.

\subsection{Mechanical properties of the matrices}

To investigate the influence on the mechanical properties of adding $\gamma$-PGA, porous and dense matrices were examined using a tensile strength instrument (Fig. 4). The maximum load of the porous matrix dose-dependently increased with the addition of $\gamma$-PGA. When comparing the $\mathrm{C} 100$ matrix with the P20C80 composite matrix, the maximum load increased by about $50 \%$, from $0.96 \mathrm{~N}$ (C100) to $1.39 \mathrm{~N}$ (P20C80) (Fig. 4). Since the matrices had been treated with $\mathrm{NaOH}$ which neutralized the acidic property of the amino groups of chitosan, we propose that the increment in the maximum load of $\gamma-\mathrm{PGA} /$ chitosan composite porous matrices was attributable to the hydrogen bonding and other forces, not to intermolecular electrostatic interactions. By increasing the amount of $\gamma$-PGA in the porous matrix, the quantity of hydrogen bonds and other interactions also increased, and thus the maximum load (i.e., the strength) of the porous matrix was larger.

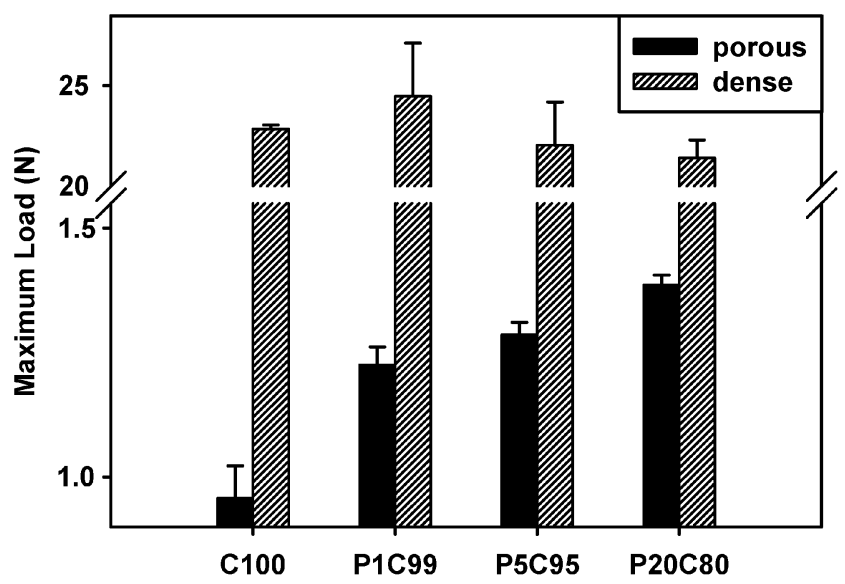

Fig. 4. Maximum loads of the porous and dense matrices. $(n=10$, mean $\pm \mathrm{SD})$.

Unlike the porous matrices, the maximum load of the dense matrices did not increase with the addition of $\gamma$-PGA (Fig. 4). To explain this phenomenon, it is helpful to compare the values of the maximum loads of the dense and porous matrices. The maximum load of the dense chitosan $(\mathrm{C} 100)$ matrix $(22.85 \mathrm{~N})$ was about 20 times that of the porous chitosan matrix $(0.96 \mathrm{~N})$. Therefore, the mechanical strength of the dense chitosan matrix was so good that the contribution of $\gamma$-PGA was relatively negligible.

\subsection{Adsorption of serum proteins onto matrix surfaces}

The adsorption of serum proteins can affect cell attachment and proliferation on matrix surfaces, an important issue in tissue engineering. As shown in Table 3, the adsorption of serum proteins increased as the amounts of $\gamma$-PGA in the composite matrices increased. The amount of serum proteins adsorbed onto the P20C80 surface was about 1.5 times that onto the C100 surface.

The reason that $\gamma$-PGA increased the adsorption of serum proteins is not clear. We propose that the hydrophilicity and high viscosity of $\gamma$-PGA may have 
Table 3

Adsorption of serum proteins on the chitosan $(\mathrm{C} 100)$ and $\gamma$-PGA/ chitosan (P1C99, P5C95, and P20C80) surfaces $(n=3$, mean \pm SD)

\begin{tabular}{lllll}
\hline & C100 & P1C99 & P5C95 & P20C80 \\
\hline $\begin{array}{l}\text { Protein adsorbed } \\
(\mu \mathrm{g})\end{array}$ & $2.06 \pm 0.17$ & $2.28 \pm 0.20$ & $2.74 \pm 0.09$ & $3.30 \pm 0.03$ \\
\hline
\end{tabular}

contributed to this effect. Other factors such as hydrogen bonds and electrostatic forces may also have played some roles.

\subsection{Cytocompatibility of the matrices}

The cytocompatibility of the matrices is very important for their tissue engineering applications. To examine the cell-matrix interactions, ROS cells were cultivated on these matrix surfaces (dense matrices) (Fig. 5). Photographs of ROS cells on day 5 were taken to show that the cell numbers increased with the amount of $\gamma$-PGA in the matrices (Fig. 5A). It was clear that ROS cells proliferated well on the P20C80 composite surface. On the other hand, the use of C100, P1C99, and P5C95 surfaces resulted in lower numbers of ROS cells. This trend was consistent with the statistical results of cell proliferation (Fig. 5B).

By comparing the data of contact angle and swelling ratio (Table 2), we found that ROS cells proliferated better on a more-hydrophilic (with a lower contact angle) surface, such as the P20C80 surface. In addition to the effect of hydrophilicity, the presence of $\gamma$-PGA in the matrix enhanced the adsorption of serum proteins including cell attachment factors which were routinely added to the culture medium (Section 3.5). Therefore, improvements in the hydrophilicity and adsorption of attachment factors are both beneficial for cell attachment and proliferation. On the other hand, we noted that the cell numbers on day 1 did not vary among samples, indicating that the cell seeding density was quite consistent.

Observation of cell morphology is a direct way to characterize the condition of cell attachment and proliferation. The morphologies of ROS cells on the P20C80 surface were quite similar to those observed on tissue culture polystyrene, which is a commercialized material for ideal proliferation of most cells (data not shown). However, the morphology of cells on the chitosan $(\mathrm{C} 100)$ surface revealed low cell numbers and the presence of many nondividing cells (Fig. 5A), suggesting that the surface of the chitosan matrix is not a good environment for cell attachment and proliferation. Taken together, based on the data of the contact angle, swelling behavior, protein adsorption, and cell proliferation and morphology, we conclude that the addition of $\gamma$-PGA to the chitosan matrix improves
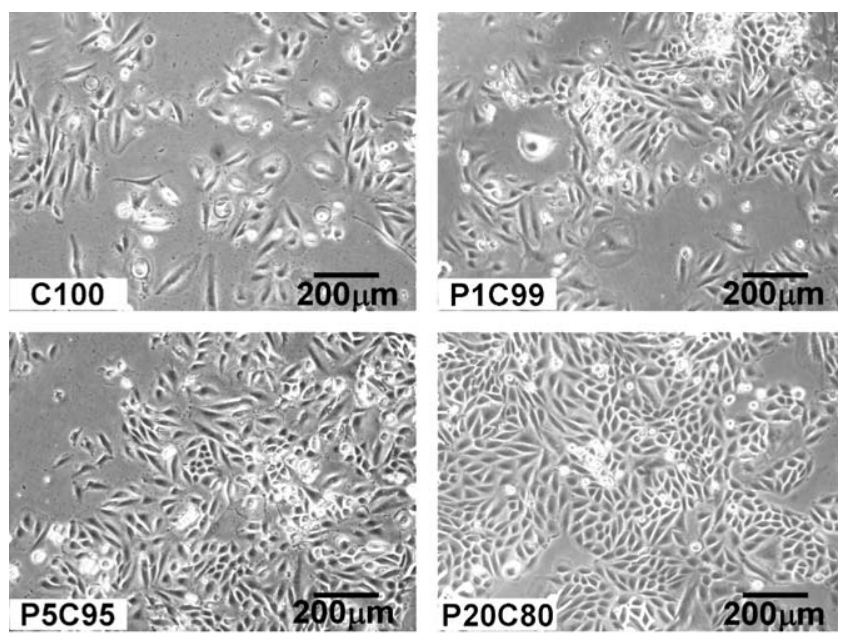

(A)

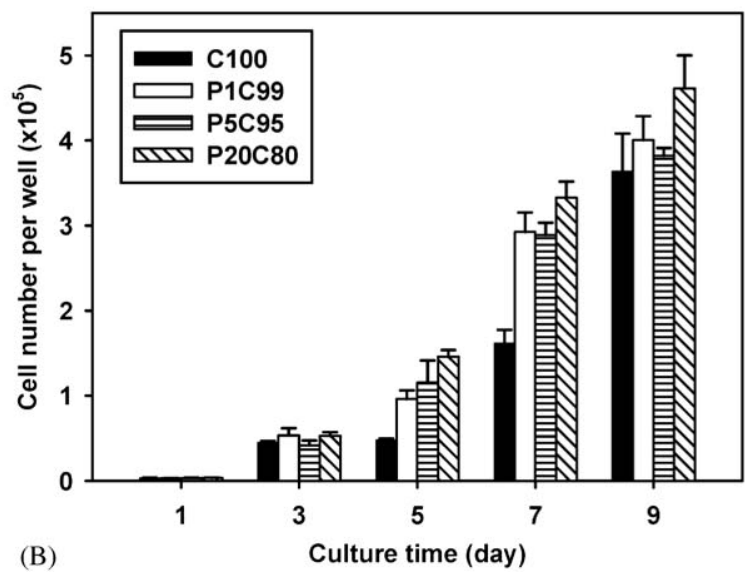

Fig. 5. (A) Optical photographs $(40 \times)$ and (B) proliferation of ROS cells cultivated on the chitosan $(\mathrm{C} 100)$ and $\gamma$-PGA/chitosan (P1C99, P5C95, and P20C80) surfaces. $(n=4$, mean $\pm \mathrm{SD})$.

the hydrophilicity, enhances serum protein adsorption, and promotes the attachment and proliferation of ROS cells on the matrix.

\section{Conclusions}

We chose $\gamma$-PGA for modifying chitosan matrices and successfully prepared a $\gamma$-PGA/chitosan composite biomaterial. In this experiment, we discovered that $\gamma$-PGA can be well mixed with chitosan to fabricate both dense and porous $\gamma$-PGA/chitosan composite matrices. The porous matrices have an interconnected pore structure with a pore size of $30-100 \mu \mathrm{m}$. The hydrophilicity and serum proteins adsorption of the composite matrices can be significantly enhanced. With respect to the mechanical properties, the addition of $\gamma$-PGA increased the maximum load (strength) of the composite matrices. Using ROS cells to examine the cytocompatibility, we found that the cells attached and proliferated 
more prosperously on the $\gamma$-PGA/chitosan composite matrices as compared with the chitosan matrix. Consequently, with the addition of $\gamma$-PGA, many properties of the chitosan matrix were modified and enhanced, making the $\gamma$-PGA/chitosan composite matrix a very promising biomaterial for tissue engineering applications.

\section{Acknowledgements}

The authors thank Ms. Su-Jen Ji and Prof. LiangPing Lin, Advanced Instrument Center, National Taiwan University, for their assistance with the SEM analyses of the matrices. This study was financially supported by the National Science Council, Taiwan (Grant numbers: NSC91-2214-E-002-035 and NSC922214-E-002-034).

\section{References}

[1] Muzzarelli RAA. Chitin, 1st ed. Oxford, New York: Pergamon Press; 1977.

[2] Chatelet C, Damour O, Domard A. Influence of the degree of acetylation on some biological properties of chitosan films. Biomaterials 2001;22(3):261-8.

[3] Madihally SV, Matthew HW. Porous chitosan scaffolds for tissue engineering. Biomaterials 1999;20(12):1133-42.

[4] Koyano T, Minoura N, Nagura M, Kobayashi K. Attachment and growth of cultured fibroblast cells on PVA/chitosan-blended hydrogels. J Biomed Mater Res 1998;39(3):486-90.

[5] Khor E, Lim LY. Implantable applications of chitin and chitosan. Biomaterials 2003;24(13):2339-49.

[6] Rhoades J, Roller S. Antimicrobial actions of degraded and native chitosan against spoilage organisms in laboratory media and foods. Appl Environ Microbiol 2000;66(1):80-6.

[7] Lee KY, Ha WS, Park WH. Blood compatibility and biodegradability of partially $N$-acylated chitosan derivatives. Biomaterials 1995;16(16):1211-6.

[8] Kratz G, Arnander C, Swedenborg J, Back M, Falk C, Gouda I, Larm O. Heparin-chitosan complexes stimulate wound healing in human skin. Scand J Plast Reconstr Surg Hand Surg 1997;31(2): 119-23.

[9] Miyazaki S, Ishii K, Nadai T. The use of chitin and chitosan as drug carriers. Chem Pharm Bull (Tokyo) 1981;29(10):3067-9.

[10] Roller S, Covill N. The antifungal properties of chitosan in laboratory media and apple juice. Int J Food Microbiol 1999;47(1-2):67-77.

[11] Kaplan D. Biopolymers from renewable resources. Berlin, New York: Springer; 1998.

[12] Iwata H, Matsuda S, Mitsuhashi K, Itoh E, Ikada Y. A novel surgical glue composed of gelatin and $N$-hydroxysuccinimide activated poly(L-glutamic acid): Part 1. Synthesis of activated poly(L-glutamic acid) and its gelation with gelatin. Biomaterials 1998;19(20):1869-76.

[13] Li C, Ke S, Wu QP, Tansey W, Hunter N, Buchmiller LM, Milas L, Charnsangavej C, Wallace S. Tumor irradiation enhances the tumor-specific distribution of poly(L-glutamic acid)-conjugated paclitaxel and its antitumor efficacy. Clin Cancer Res 2000;6(7): 2829-34.

[14] Multani AS, Li C, Ozen M, Yadav M, Yu DF, Wallace S, Pathak S. Paclitaxel and water-soluble poly(L-glutamic acid)-paclitaxel, induce direct chromosomal abnormalities and cell death in a murine metastatic melanoma cell line. Anticancer Res 1997;17(6D):4269-74.

[15] Otani Y, Tabata Y, Ikada Y. A new biological glue from gelatin and poly(L-glutamic acid). J Biomed Mater Res 1996;31(2): 158-66.

[16] Otani Y, Tabata Y, Ikada Y. Sealing effect of rapidly curable gelatin-poly(L-glutamic acid) hydrogel glue on lung air leak. Ann Thorac Surg 1999;67(4):922-6.

[17] Richard A, Margaritis A. Poly(glutamic acid) for biomedical applications. Crit Rev Biotechnol 2001;21(4):219-32.

[18] Staikos G, Bokias G, Tsitsilianis C. The viscometric methods in the investigation of the polyacid polybase interpolymer complexes. J Appl Polym Sci 1993;48(2):215-7.

[19] Lysenko EA, Bronich TK, Eisenberg A, Kabanov VA, Kabanov AV. Block ionomer complexes from polystyrene-block-polyacrylate anions and $N$-cetylpyridinium cations. Macromolecules 1998;31(14):4511-5.

[20] Sakiyama T, Chu CH, Fujii T, Yano T. Preparation of a polyelectrolyte complex gel from chitosan and kappa-carrageenan and its pH-sensitive swelling. J Appl Polym Sci 1993;50(11): 2021-5.

[21] Chilvers GR, Morris VJ. Coacervation of gelatin gellan gum mixtures and their use in microencapsulation. Carbohyd Polym 1987;7(2):111-20.

[22] Yamamoto H, Amaike M. Biodegradation of cross-linked chitosan gels by a microorganism. Macromolecules 1997; 30(13):3936-7.

[23] Yamamoto H, Horita C, Senoo Y, Nishida A, Ohkawa K. Polyion complex fiber and capsule formed by self-assembly of poly-L-lysine and gellan at solution interfaces. J Appl Polym Sci 2001;79(3):437-46.

[24] Yamamoto H, Senoo Y. Polyion complex fiber and capsule formed by self-assembly of chitosan and gellan at solution interfaces. Macromol Chem Phys 2000;201(1):84-92.

[25] Amaike M, Senoo Y, Yamamoto H. Sphere, honeycomb, regularly spaced droplet and fiber structures of polyion complexes of chitosan and gellan. Macromol Rapid Commun 1998; 19(6):287-9.

[26] Ohkawa K, Kitsuki T, Amaike M, Saitoh H, Yamamoto H. Biodegradation of ornithine-containing polylysine hydrogels. Biomaterials 1998;19(20):1855-60.

[27] Ohkawa K, Takahashi Y, Yamada M, Yamamoto H. Polyion complex fiber and capsule formed by self-assembly of chitosan and poly(alpha,L-glutamic acid) at solution interfaces. Macromol Mater Eng 2001;286(3):168-75.

[28] Ho MH, Kuo PY, Hsieh HJ, Hsien TY, Hou LT, Lai JY, Wang DM. Preparation of porous scaffolds by using freeze-extraction and freeze-gelation methods. Biomaterials 2004;25(1):129-38.

[29] The TH, Feltkamp TE. Conjugation of fluorescein isothiocyanate to antibodies. I. Experiments on the conditions of conjugation. Immunology 1970;18(6):865-73.

[30] The TH, Feltkamp TE. Conjugation of fluorescein isothiocyanate to antibodies. II. A reproducible method. Immunology 1970;18(6):875-81.

[31] Olde Damink LH, Dijkstra PJ, van Luyn MJ, van Wachem PB, Nieuwenhuis P, Feijen J. Cross-linking of dermal sheep collagen using a water-soluble carbodiimide. Biomaterials 1996;17(8): 765-73. 